\title{
Primate Sleep in Phylogenetic Perspective
}

\section{Citation}

Nunn, Charles Lindsay, Patrick McNamara, Isabella Capellini, Brian T. Preston, and Robert A. Barton. 2010. Primate sleep in phylogenetic perspective. In Evolution of sleep: Phylogenetic and functional perspectives, ed. P. McNamara, R.A. Barton and C.L. Nunn. Cambridge, UK; New York: Cambridge University Press.

\section{Permanent link}

http://nrs.harvard.edu/urn-3:HUL.InstRepos:4340687

\section{Terms of Use}

This article was downloaded from Harvard University's DASH repository, and is made available under the terms and conditions applicable to Other Posted Material, as set forth at http:// nrs.harvard.edu/urn-3:HUL.InstRepos:dash.current.terms-of-use\#LAA

\section{Share Your Story}

The Harvard community has made this article openly available.

Please share how this access benefits you. Submit a story.

Accessibility 


\section{Primate Sleep in Phylogenetic Perspective}

Charles L. Nunn, Patrick McNamara, Isabella Capellini, Brian T. Preston and Robert A. Barton

$\dagger$ Author Note. This material is based upon work supported, in part, by the National Institute of Mental Health, Grant 5R01MH070415-02 


\section{Introduction}

The Primates are a diverse group of eutherian mammals, with between about 200 and 400 species, depending on the taxonomic authority that is consulted (e.g., Corbet \& Hill, 1991; Wilson \& Reeder, 2005). Most primate species dwell in tropical forests, but primates also thrive in many other habitats, including savannas, mountainous forests of China and Japan, and even some urban areas. Living primates are divided into two groups, the Strepsirhini (lemurs and lorises) and the Haplorhini (monkeys, apes and the tarsier). Strepsirhines include mostly arboreal species and retain several ancestral characteristics, including greater reliance on smell and (in most species) a dental comb that is used for grooming. Most are nocturnal, but some have, in parallel with most haplorhines, evolved a diurnal niche. They are found only in the Old World tropics. Haplorhines are more widely distributed geographically, being found in both the New and Old World. Haplorhines include two groups, the Platyrrhini and the Catarrhini. Platyrrhines are monkeys that are native to the New World. Catarrhines include both Old World monkeys and the apes. With the exception of owl monkeys in the genus Aotus, all monkeys and apes are active during the day (i.e., diurnal), and most live in bisexual social groups that vary in size from two to well over 100 adults (Smuts, Cheney, Seyfarth, Wrangham, \& Struhsaker, 1987).

Nonhuman primates are among the best studied of mammals, in large part because of their close phylogenetic relatedness to humans. Much of the research on wild primates has focused on issues of biomedical importance, such as emerging infectious diseases (Chapman, Gillespie, \& Goldberg, 2005; Wolfe, et al., 1998). As a result, we know much about the parasites and pathogens that infect wild primates (Nunn \& Altizer, 2005, 2006). An additional goal of studying primates is to gain insight into human evolution (Foley \& Lee, 1989; Smuts et al., 1987). Thus, a wealth of information is available on primate behavior and ecology (Smuts et al., 1987), primate phylogeny (Disotell, 2008; Purvis, 1995), and the geographical distribution and population sizes of different primate species (Hilton-Taylor, 2002). 
Sleep is an important factor in non-human primate health, behavior and ecology and can play a central role in shaping daily activity schedules (Anderson, 1998, 2000). For instance, locating a suitable sleep site can be an important component of individual survival in primates, allowing them to avoid mosquito vectors or predators (Anderson, 1998, 2000; Day \& Elwood, 1999; Di Bitetti, Vidal, Baldovino, \& Benesovsky, 2000; Heymann, 1995; Nunn \& Heymann, 2005). Thus, Hamadryas baboons (Papio hamadryas) travel to cliffs to sleep at night (Kummer, 1968), while chimpanzees (Pan troglodytes) build a new nest every night for sleeping (Boesch \& Boesch-Achermann, 2000).

Together, these factors make primates particularly valuable for the comparative study of sleep, with the potential to provide critical advances in our understanding of human sleep disorders, the ecology of sleep in non-human primates and humans, and the evolution of sleep patterns more generally. Properly controlled comparative studies of sleep have been rare in primates and other mammals, usually requiring comparative biologists to examine variation across mammals rather than within different orders of mammals (Capellini, Barton, McNamara, Preston, \& Nunn, 2008; Capellini, Nunn, McNamara, Preston, \& Barton, 2008; Elgar, Pagel, \& Harvey, 1988; Lesku, Roth, Amlaner, \& Lima, 2006). A handful of studies have investigated the durations of REM and NREM sleep in primates, however, providing some data for at least initial comparative studies of primate sleep (e.g., Bert \& Pegram, 1969; Hsieh, Robinson, \& Fuller, 2008; Perachio, 1970).

In this chapter, we review existing knowledge of sleep in primates, focusing in particular on variation in sleep quotas across primate species in relation to ecological and life history traits. Our goals are threefold. First, we aim to identify those aspects of sleep that have changed on the primate lineage. Second, based on our review, we advance selected hypotheses for distinctive characteristics of sleep expression in primates. We test these hypotheses when sufficient data 
exist. Lastly, we summarize gaps in our knowledge of primate sleep, and we identify the primate species that are most important for future data collection. We propose more generally that increased knowledge of sleep expression among non-human primates will deepen our understanding of the function of sleep and human sleep disorders.

\section{The measurement of primate sleep quotas and sleep architecture}

We focus much of this review on four basic parameters of sleep expression in primates that have become known as "sleep quotas." These simply represent the total time spent asleep per day and the time spent in each of the two major forms of mammalian sleep: active or REM (rapid eye movement) sleep, and quiet or non-REM (NREM) sleep. Periods of REM and NREM sleep alternate through the sleeping period, and the mean duration of these sleep cycles - measured from initiation of NREM to the end of the subsequent bout of REM sleep - is the fourth parameter of interest.

Despite the apparent validity of using sleep quotas to study sleep expression across primates, these data must be interpreted with caution given several methodological problems associated with the collection of sleep quotas. Among the most troublesome of these issues has been the necessity for animals to be studied in the laboratory rather than in their natural habitats. When studied in the laboratory, animals may be restrained in order to record EEG sleep changes. Restraint for animals can be very stressful, and this could affect the sleep data obtained from the experiment. Data from telemetric recordings are available for only a handful of primates, including baboons (Bert, 1975), lemurs (Vuillon-Cacciuttolo, Balzamo, Petter, \& Bert, 1976), monkeys (Hsieh et al., 2008; Reite, Stynes, Vaughn, Pauley \& Short, 1976), and chimpanzees (Bert, Kripke, \& Rhodes, 1970), and few would consider these studies to be "natural" with respect to the behavior and ecology of the animals involved. In these studies, the animals undergo surgery to implant electrodes that can measure brain wave activity and transmit the 
information to a receiver. The transmitter was usually housed in a small box that was affixed to the top of the animal's head, allowing the animal the freedom to move without attached wires. Twenty-four hour EEG recordings could therefore be obtained from the animal while it moved about and while it slept. There was no need to strap the animal into a chair or restraining device, or to drug the animal so that EEG recordings could be secured. In that sense the animal was not restrained when telemetry was used. Recent advances in EEG data acquisition technology offer many promising opportunities for studies of sleep in wild, naturally behaving primates (Rattenborg et al., 2008).

Other factors may also influence sleep measures. For example, the ambient temperature in a laboratory setting may differ from conditions typical in the wild; this is relevant because sleep variables are known to be sensitive to small temperature changes. Usually laboratories are under constant lighting, which is likely to impact components of the sleep response in some animals. Thus, comparative biologists should restrict their analysis to sleep studies that meet a set of basic criteria (e.g., Capellini, Barton et al., 2008; McNamara, Capellini, Harris, Nunn, Barton, \& Preston, 2008). Specifically, the ideal should be to use data that record the animal's brain activity with an electroencephalogram (EEG) for at least 24 hours under normal (for the animal) light-dark schedules and ambient temperatures, and only after the animal has adapted to the laboratory and recording procedures. Unfortunately, we cannot yet meet this ideal for comparative studies of primates. In this chapter, we therefore included behavioral measures of sleep for some estimates of total sleep times, and limited the data to studies with 12 or more hours of observation (which should be sufficient for most species of primates that sleep in only one block of time per day, i.e. they are monophasic).

Empirical data and general evolutionary patterns. Available data on average sleep quotas in primates are provided in Table 1 (McNamara et al., 2008). We found data on 20 species of nonhuman primates, to which we also added data on humans from Carskadon and Dement (2006). Owl monkeys, cotton top tamarins (Saguinus oedipus) and mouse lemurs 
(Microcebus murinus) appear to be the "marathon sleepers" among the primate species studied thus far. Their average total sleep time per day ranges from 13 to 17 hours. Interestingly, the two longest-sleeping species are nocturnal (owl monkeys and mouse lemurs), a topic that we turn to below. Our evolutionarily closest relative, the chimpanzee, sleeps an average of 11.5 hours per day, quite close to the phylogenetic average for all primates. The short sleepers in our data set sleep for 8-10 hours and are phylogenetically diverse; they include humans, a handful of cercopithecine monkeys, a lemur, and some New World primates.

With these data, we can investigate evolutionary patterns, for example by reconstructing ancestral states of sleep traits on primate phylogeny. Before conducting such tests, however, it is important to assess whether primate sleep quotas exhibit what evolutionary biologists call "phylogenetic signal" (Blomberg \& Garland, 2002; Blomberg, Garland, \& Ives, 2003). This concept simply captures whether more closely related species exhibit more similar values in their sleep quotas; such an effect would indicate that the trait is shared through common descent, suggesting that it is an evolved trait. In addition, phylogenetic signal would show that data vary sufficiently across species for comparative study, such that measurements available for different species can be linked to ecological, life history or behavioral traits of those species. Phylogenetic signal in sleep traits has been shown to exist across a wide range of mammalian species (Capellini, Barton et al., 2008), but this appears to be mostly structured at the order level - e.g. with rodents exhibiting more similarity to other rodents than to carnivores - and we lack an understanding of patterns of phylogenetic signal within orders, including primates.

Figures 1-3 indicate that more closely related primate species exhibit similar trait values, and a statistical test for phylogenetic signal (Blomberg et al., 2003) reveals some evidence for greater similarity among more closely related primate species, especially for REM and NREM sleep durations examined separately (Table 2). Although these test results are not statistically significant, they approach significance, and given the small sample sizes and the probable measurement error involved with estimating sleep quotas, it is reasonable to suggest that these 
results point towards the existence of phylogenetic signal (Blomberg et al., 2003; Ives, Midford, \& Garland, 2007). These analyses thus suggest that evolutionary history explains some of the variation in primate sleep traits, as was found more convincingly in a larger set of mammals (Capellini, Barton, et al., 2008).

Assuming that evidence for phylogenetic signal is strengthened as more species of primates are studied, we can also use phylogenetic methods to reconstruct sleep characteristics of the ancestral primate and to examine trends in sleep characteristics over primate evolution. To reconstruct sleep in the ancestral primate, we used a Bayesian approach, as this provides a means to put confidence intervals on the reconstructed ancestral node (Pagel, Meade, \& Barker, 2004). To implement these tests, we used the program BayesTraits (Pagel \& Meade, 2007) with Purvis' (1995) "supertree" for the estimate of primate phylogeny. From this analysis, we estimate that the ancestral primate slept an average of just over 11 hours per day, with 10 hours of NREM and 1.3 hours of REM sleep. The confidence intervals are provided in Table 3 .

This methodology also provides another way to assess phylogenetic signal (Freckleton, Harvey, \& Pagel, 2002) and models of evolutionary change (Pagel, 1997, 1999). For example, the program BayesTraits calculates a parameter known as $\lambda$, ranging between 0 (no phylogenetic signal) and 1 (phylogenetic signal consistent with a Brownian motion model of evolution); higher values thus indicate greater phylogenetic signal (Freckleton et al., 2002). The values presented in Table 3 suggest that REM sleep exhibits more phylogenetic signal than other traits. Conversely, the parameter $\kappa$ investigates how evolutionary rate varies in relation to branch length (i.e., the time separating speciation events on the phylogeny). We find that $\kappa$ is lower for REM than for NREM. This indicates that especially for NREM sleep, more change in sleep times occurs on longer branches, thus suggesting that sleep has not evolved according to an adaptive radiation model (with large changes early in a clade and occurring on short branches). Similarly, the values of $\delta>1$ indicate that more change occurs later in evolution than in the early stages and confirms a pattern of species-adaptation rather than of early adaptive radiation. From this 
analysis, it appears that the two forms of sleep have undergone somewhat different evolutionary trajectories in primates. We should note, however, that most of these parameters had extremely wide confidence intervals that encompassed one, probably due to the small sample sizes, and thus we consider these analyses to be exploratory.

Primates also exhibit variability in the amount of time devoted to NREM sleep and REM sleep (Table 1). For example, monkeys spend between 7 and 15 hours in NREM sleep (Figure 2). Time devoted to REM sleep varies from a little over 30 minutes per day in the vervet monkey (Cercopithecus aethiops) to 2 hours per day in the chimpanzee and human (Figure 3). REM sleep may have increased in the great apes, although this is based on only two ape species in the data set, and increases in other lineages as can be seen in Figure 3.

In general, it appears that total sleep duration in primates is most sensitive to the amount of NREM sleep. Thus, in analyses of independent contrasts that control for the nonindependence of species values (Felsenstein, 1985; Garland, Harvey, \& Ives, 1992; Nunn \& Barton, 2001), we found that evolutionary increases in NREM sleep correlate strongly with evolutionary increases in total sleep among primates, while REM sleep shows no such association (Figure 4, panels a and b). In contrast to work across mammals more generally (Capellini et al., 2008a), we failed to find a significant association between NREM and REM sleep in primates (Figure 4.c). While this may again reflect low statistical power, it is noteworthy that the slope of this non-significant relationship is in fact negative (see Figure 4 legend), whereas previous work demonstrated positive associations across mammals more generally (Capellini et al., 2008a). Additional data collection on primate sleep may reveal a difference in this regard in primates compared to other mammals. 


\section{Sleep in relation to biological characteristics of primates}

In the previous section we showed how a species' evolutionary history helps explain why closely related species have similar sleep durations, but what explains the remaining variation in primate sleep? Here, we consider factors that might account for variability in sleep patterns. In this section, we first review features of primates that might be related to sleep characteristics, and then perform tests to investigate at least some of these predictions when sufficient data exist.

It is generally agreed that one of the major evolutionary transitions in the primate order involved a shift from a nocturnal to a diurnal activity period, which has occurred more than once (Martin, 1990, \#1703). The shift from nocturnality to diurnality was associated with dramatic changes in ecology and behavioral capacities. Prominent among ecological changes was increased predation pressure from diurnal predators, such as raptors, leading to a requirement to visually monitor the environment for these and other predators. The shift to a diurnal lifestyle also may have played a role in the evolution of larger, permanent social groups in most primate lineages, as this would have afforded greater safety from predators (Janson, 1992; van Schaik, 1983). Moreover, some diurnal lineages became more terrestrial and moved into more open habitats (Nunn \& Barton, 2001). Living on the ground may have exposed these animals to greater predation pressure, and thus selection for even larger social groups (Nunn \& van Schaik, 2002). Finally, the shift to diurnality is associated with greater sexual selection, especially among terrestrial species (e.g., greater body mass dimorphism, Plavcan \& van Schaik, 1997).

In terms of brain evolution, there emerged a tendency among diurnal primates towards reduction of the olfactory region of the brain and expansion of the cerebral cortex associated with an increasing reliance on visual sensory modalities (Barton, Purvis, \& Harvey, 1995). Diurnal primates, for example, have either dichromatic or trichromatic color vision and fields of view that significantly overlap, resulting in true three-dimensional depth perception (Martin \& Ross, 2005, \#10941; Cartmill, 1974,\#10940). The combination of greater predation pressures and enhanced social interaction likely also promoted expansions in brain volumes linked to 
visual processing and management of social capacities (Barton, 1998; Barton \& Dunbar, 1997). In primates, living in a larger social group is associated with increased neocortex size (Dunbar, 1992; 1998), and increasing sexual selection covaries with the size of brain structures involved in sensory-motor skills (Lindenfors, Nunn, \& Barton, 2007).

The evolutionary shift to a diurnal lifestyle, therefore, had an enormous impact on primate behavior and life history strategies. In what follows, we consider how these and other features may have influenced primate sleep. When possible, we test these proposals using data from The Phylogeny of Sleep research group (McNamara et al., 2008).

1) Evolution of monophasic sleep. One of the most fundamental descriptors of sleep concerns whether it occurs in one bout per 24-hour period of time (i.e., monophasic), or whether it encompasses multiple bouts in a daily cycle (polyphasic, Ball, 1992). The occurrence of sleep relative to the daily photoperiod varies across mammals, with most species exhibiting a polyphasic form of sleep characterized by short bouts of sleep throughout the day and night. This polyphasic pattern is most likely to be the ancestral state in mammals, based on a maximum likelihood analysis of 56 species (Capellini et al., 2008b). Some lemur species, such as Eulemur, show an interesting pattern of cathemerality, meaning that they are active during both day and night (Tattersall, 1987). Sleep is not so rigidly restricted to night or day in these species, potentially resulting in a tendency towards polyphasic sleep patterns, and the same appears to be true of the nocturnal mouse lemur (Capellini et al., 2008b). In anthropoid primates, however, sleep is clearly monophasic, with one sleep period during the 24-hour cycle (Capellini et al., 2008b).

Thus, we conclude that monophasic sleep is a derived trait in anthropoid primates, with an origin either at the base of the primate clade (with subsequent reversals in some strepsirhines), or probably originating on the lineage leading to monkeys and apes.

2) Reductions in sleep among diurnal primates. Our analyses on the phylogenetic distribution of total sleep, NREM sleep and REM sleep suggests that diurnal activity period 
exerted a major influence on patterns of sleep in primates, with diurnal primates sleeping less than nocturnal primates. Total sleep duration appears to vary according to whether the species is nocturnal or diurnal, with longer sleep durations in nocturnal primate species (see Figure 1). This could reflect that many small-bodied nocturnal species, such as the mouse lemur, seek protected sleep sites to reduce predation pressure, which in turn allows them to sleep for longer. Conversely, the shorter sleep durations in diurnal species may reflect increased sleep efficiency associated with monophasic sleep (see below), increased foraging needs that limit the time available for sleep, or theromoregulatory costs associated with inactivity during the night, when temperatures are at the lowest. In addition, the increased needs for social interactions in these social species may also constrain time available for sleeping.

We examined these patterns using phylogenetically-based statistical methods. Among the species in our data set, total sleep time decreases over the three independent transitions in activity period from nocturnality to diurnality. When treating activity period as a continuously varying character, the association between diurnality and total sleep time is significant $\left(\mathrm{t}_{17}=-2.94\right.$, $\mathrm{P}=0.009$ ), although based on only five informative contrasts (i.e., the others exhibit no variation in the activity period variable). Some phylogenetic assumptions are violated in this analysis, likely due to treating activity period as if it is a continuous character. Further information on sleep in other lineages of primates - as well as other mammals - would help to address the effects of nocturnality more directly .

3) Increased sleep intensity. Primate sleep exhibits a differentiation of NREM sleep into at least two distinctive types: a light form characterized by spindling activity, and a deep form characterized by slow wave activity $\{$ SWA; Balzamo, Santucci, Seri, Vuillon-Cacciuttolo, \& Bert, 1977 \#10900; Hsieh et al., 2008 \#10901\}. In the great apes (including humans), NREM sleep can be differentiated into four sub-stages, with sub-stages III and IV marked predominantly by SWA or delta activity, which indexes sleep intensity in humans (Tobler, 2005). Stages I and II are characterized mainly by spindling activity. Interestingly, while in most primates spindling 
activity is more characteristic of juvenile than of adult sleep, marked spindling activity appears to persist into the adult state in the great apes, including humans (Bert, Balzamo, Chase, \& Pegram, 1975). The differentiation of NREM into sub-stages with their concomitant spindling activity and greater SWA may have helped primates to achieve enhanced sleep intensity for each bout of sleep. Indeed, monophasic sleep in mammals may be more efficient because it involves less time in light sleep and monophasic sleepers spend less time asleep per day (Capellini et al., 2008b).

On the other hand, it could also be that nocturnal predation risk on diurnal primates favored lighter sleep, as a means to detect predators (Lima, Rattenborg, Lesku, \& Amlaner, 2005). Lighter sleep might also be favored in the context of social sleeping, for example to monitor competitive interactions, mating opportunities, or risks to infants from other individuals in the group (especially male infanticide, van Schaik \& Janson, 2000).

4) Altered developmental sleep patterns. The shift to a diurnal activity pattern exposed primates to a different suite of predation pressures. ith increased predation, one might expect that primate young would be born in a precocial state. Perhaps consistent with this prediction, we see a transition from "parking" infants in a safe place among some strepsirrhines, to being carried by the mother in haplorrhines. REM sleep has been implicated in brain development owing to the age-related changes in its expression, with REM sleep dominating the sleep of juveniles (Reite et al., 1976, \#9029; Carroll, Denenberg, \& Thoman, 1999,\#10902). In addition, previous comparative analyses have suggested that mammalian species that give birth to immature (altricial) infants - those requiring the highest degree of subsequent brain development - exhibit longer durations of REM sleep (Zepelin, Seigel, \& Tobler, 2005). However, a recent study called into question the generality of this result in phylogeny-based tests (Capellini et al., 2008a, \#10532), as did a more focused study of three mammalian species (Thurber, Jha, Coleman, \& Frank, 2008). Thurber et al. showed that when sleep times are compared at a common developmental landmark (age at eyes opening), ferret kits - considered among the most 
altricial neonates among mammals - do not show longer sleep times than other species (cats and rats), as predicted by the hypothesis that REM sleep helps developing the neonatal brain. In addition, ferrets have less REM sleep (55\% of total sleep time) than the other two species (75\% in both cats and rats).

Nonetheless, primate juveniles may devote less of their sleep time to REM, as compared to other mammalian juveniles, and in accordance with this general association between REM and altriciality. According to Bert and colleagues (Bert, 1971; Bert, Pegram, \& Balzamo, 1972), the amount of time spent in REM is reduced in juvenile primates relative to other mammals. For example, vervet monkey juveniles spend $5.6 \%$ of their sleep time in REM; patas monkey $7.9 \%$; wild baboons, 5.9\%; Macaca radiata 11.5\%, Macaca nemestrina 11.1\%; and Macaca mulatta $15.5 \%$. This overall primate trend in reduction of juvenile REM sleep quotas is partially reversed in chimpanzees and humans, where infants spend between 22 and 50\% of their time in REM (Balzamo, Bradley, \& Rhodes, 1972 \#10904; Balzamo et al., 1972 \#10903; Salzarulo \& Ficca, 2002 \#10905). This could perhaps reflect their relatively more altricial state at birth (at least for humans), or it could reflect differences in cognitive demands in these species and the need for investment in brain tissue.

5) Sociality and primate sleep. Increased predation pressures and sociality in general may also promote the practice of co-sleeping between mother and infant, and sleeping in "huddles" with kin and non-kin in the social group. The primate infant extracts metabolic resources from the mother throughout the night, and co-sleeping could protect infants from competitive interactions within groups, including infanticide attempts by males. Koyama (1973) and Vessey (1973) described sleeping huddles in free-ranging bonnet (M. radiata) and rhesus macaques (M. mulatta), respectively. In both species, the most frequent huddle size was two, and huddles were composed primarily of mother-infant pairs, same-sex individuals, or malefemale sexual consortships. The co-sleeping pattern is established in infancy but persists into adulthood. In infancy, co-sleeping involves nursing during the night. Juveniles who are weaned 
regularly return to sleep in contact with the mother at night (e.g., baboons: Altmann, Altmann, \& Hausfater, 1981; gorillas: Goodall, 1979; orangutans: Horr, 1977).

In addition to protection against predation, the practice of the sleep huddle or co-sleeping among adults may also serve other functions. Some of these functions may include a thermoregulatory function (e.g., Altmann, 1980; Gartlan \& Brain, 1968; Suzuki, 1965; Gaulin \& Gaulin, 1982; Anderson \& McGrew, 1984) or a sexual function (Anderson \& McGrew, 1984; Fruth \& Hohmann, 1993). It is also known, however, that sleeping in a larger group might increase the risk of acquiring vector-borne infections, particularly malaria. Thus, in New World primates, the prevalence of malaria increases when the number of animals sleeping in a group increases (Figure 5, Davies, Ayres, Dye, \& Deane, 1991; Nunn \& Heymann, 2005). This probably reflects that larger groups of animals emit more of the cues used by mosquitoes to locate hosts, and it suggests that sleeping in larger groups and re-use of sleep sites (Hausfater \& Meade, 1982) might have costs to primates (Nunn \& Altizer, 2006).

An intriguing topic for the future concerns the possibility of links between sociality and sleep quotas. A recent study of Drosophila found that when flies lived in socially enriched environments with many conspecifics, they exhibited increased sleep times (Ganguly-Fitzgerald, Donlea, \& Shaw, 2006). This increased sleep time affected daytime but not nighttime sleep. A previous study conducted across mammals found that increased social sleeping correlates with shorter sleep durations (Capellini et al., 2008a). This could indicate the existence of tradeoffs between time for social interactions and sleep, or it could reflect increased sleep efficiency in social species, possibly because they gain safety in numbers and can thus spend more time in deep sleep, but these two hypothses are not mutually exclusive. We tested whether primates that live in larger networks of other individuals sleep for a longer period of time each day, but we found no significant association (independent contrasts: $t_{18}=-0.13, P=0.90$; humans were removed from the analysis due to uncertainty in measuring group size). Other measures of 
sociality, such as the time spent grooming or the number of grooming partners, would be interesting to examine in future research.

\section{Conclusions and Recommendations for Future Research}

Based on a review of the existing literature and new analyses conducted for this chapter, we identified five major hypothesized characteristics of primate sleep, many of which were associated with transitions to increased diurnality. These characteristics include the following: (1) Consolidation of sleep into a single long bout, possibly to achieve greater sleep intensities, but this also could be a side effect of typically strict activity periods (nocturnal vs. diurnal lifestyles). (2) Reductions in sleep times among diurnal primate species, which could reflect a number of different advantages or constraints associated with diurnality. (3) Increased sleep intensity, possibly associated with differentiation of NREM sleep stages into lighter and deeper stages of sleep and testable once more data have accumulated on sleep intensity measures. (4) Developmental shifts in sleeping patterns, including less REM among juveniles. (5) Maintenance of social contact during sleep, which likely has advantages in terms of infant care, predation risk and thermoregulation, but also costs in terms of parasitism (Nunn \& Altizer, 2006).

Even when data are available to test these hypotheses, our conclusions must remain tentative given the small number of primate species studied by sleep scientists. In many ways, we see this chapter as an illustration of the questions that remain to be answered, and of the phylogenetic approaches that can be brought to bear on these questions. In this context, it instructive to point out the species and clades that are missing and would be most important to include in future comparative research. First, we have information on only one species of nonhuman ape - the chimpanzee. A high priority for future research should be to collect sleep data in the other great apes, specifically gorillas (Gorilla gorilla), orangutans (Pongo pygmaeus) and bonobos (Pan paniscus), along with one or more species of gibbons (Hylobates spp.). 
Second, very few diurnal strepsirhines have been sampled, and only two have been sampled for REM and NREM sleep (one nocturnal and one diurnal species). Key species to test in this regard are those with good behavioral and ecological sampling, including the ringtailed lemur (Lemur catta), sifaka (Propithecus spp.), and the brown lemur (Eulemur fulvus).

Third, among the monkeys, notable sampling gaps include the diurnal Cebidae in the New World (especially Alouatta, Cebus, Callicebus and Ateles), and the colobines in the Old World (especially one or more species of Presbytis and Colobus monkeys). In addition, while many studies have investigated sleep in macaques (Macaca), no species of arboreal guenons (Cercopithecus) have been studied for their sleep. Morevoer, Cercocebus and Mandrillus are also missing from our understanding of primate sleep, and folivores (leaf-eaters) are largely absent. Obtaining data on folivores will be critically important for understanding the links between diet, metabolic rate and sleep patterns. A comparative study (Korstjens, Lehmann, \& Dunbar, in review \#10942) has shown that folivorous primates spend more time resting (thus including both sleep and quiet resting time) relative to frugivorous species, but it remains to be clarified if such a pattern affects sleep time or only quiet resting time, and the factors that drive these differences (e.g., more time needed to find fruits or more constraints due to digestion). As noted above, it would help to have better sampling of nocturnal primates. However, given the small number of transitions in activity period in primates, it may be necessary to investigate the effect of activity period across mammals more generally.

Figure 6 reveals that sampling has occurred at a generally "shallower" depth in Old World monkeys, meaning that taxa with sleep data tend to share a common ancestor more recently, as compared to sampling for sleep in New World monkeys and strepsirhines. This might be due to higher rates of diversification in these lineages (Purvis et al., 1995), but also indicates somewhat broader phylogenetic sampling, in terms of longer phylogenetic branches covered, for all groups except Old World monkeys. All of the previous points essentially relate to aspects of the sampling "depth" shown in Figure 6. 
In summary, the evolution of primate sleep patterns are as yet little understood, with many sampling gaps related to the phylogenetic distribution of primates and the ecological characteristics that are important for testing hypotheses for the function of sleep. Nonetheless, it is possible to formulate clear hypotheses for how major transitions associated with primate evolution have impacted primate sleep patterns, and in some cases to test these hypotheses using existing data. Given the scientific and health benefits of studying primate sleep, it is critically important to fill some of the gaps in our knowledge of primate sleep, and to do so in a way that provides the strongest tests of comparative hypotheses. 


\section{References}

Altmann, J. (1980). Baboon mothers and infants. Cambridge, MA: Harvard University Press.

Altmann, J., Altmann, S., \& Hausfater, G. (1981). Physical maturation and age estimates of yellow baboons, Papio cynocephalus, in Amboseli National Park. American Journal of Primatology, 1, 389-399.

Anderson, J. R. (1998). Sleep, sleeping sites, and sleep-related activities: Awakening to their significance. American Journal of Primatology, 46, 63- 75.

Anderson, J. R. (2000). Sleep-related behavioural adaptations in free-ranging anthropoid primates. Sleep Medicine Reviews 4, 355- 373.

Anderson, J. R., \& McGrew, W. C. (1984). Guinea baboons (Papio papio) at a sleeping site. American Journal of Primatology, 6, 1-14.

Ball, N. J. (1992). The phasing of sleep in mammals. In C. Stampi (Ed.), Why we nap: Evolution, chronobiology and functions of polyphasic and ultrashort sleep (pp. 31-49). Boston: Birkhauser.

Balzamo, E., Bradley, R.J., \& Rhodes, J.M. (1972). Sleep ontogeny in the chimpanzee: From two months to forty-one months. Electroencephalography and Clinical Neurophysiology, 33, 47-60.

Balzamo, E., Santucci, V., Seri, B., Vuillon-Cacciuttolo, G., \& Bert, J. (1977). NonhumanPrimates: Laboratory animals of choice for neurophysiologic studies of sleep. Laboratory Animal Science, 27, 879-886.

Barton, R. A. (1998). Visual specialization and brain evolution in primates. The Royal Society of London Series B-Biological Sciences, 265, 1933-1937. 
Barton, R. A., \& Dunbar, R. I. M. (1997). Evolution of the social brain. In A. Whiten \& R. W. Byrne (Eds.), Machiavellian Intelligence II: Extensions and Evalutations (pp. 240-263). Cambridge: Cambridge University Press.

Barton, R. A., Purvis, A., \& Harvey, P. H. (1995). Evolutionary radiation of visual and olfactory brain systems in primates, bats and insectivores. Philosophical Transactions of the Royal Society, London, Series B, 348, 381-392.

Bert, J. (1975). Generic characteristics and specific characteristics of the ponto-geniculooccipital spike activity (PGO) in 2 baboons, Papio hamadryas and Papio papio. Brain Research, 88(2), 362-366.

Bert, J., Balzamo, E., Chase, M., \& Pegram, V. (1975). The sleep of the baboon, Papio papio, under natural conditions and in the laboratory. Electroencephalography and Clinical Neurophysiology, 39, 657-662.

Bert, J., Kripke, D. F., \& Rhodes, J. (1970). Electroencephalogram of the mature chimpanzee: Twenty-four hour recordings. Electroencephalography and Clinical Neurophysiology, $28(4), 368-373$.

Bert, J., \& Pegram, V. (1969). The sleep electroencephalogram in Cercopithecinae: Erythrocerbus patas and Cercopithecus aethiops sabaeus. Folia Primatologica, 11(1), 151-159.

Bert, J., Pegram, V, \& Balzamo, E. (1972). Comparison of sleep between 2 Macaca species (Macaca radiata and Macaca mulatta). Folia Primatologica, 17(3), 202-208. 
Blomberg, S. P., \& Garland, T. (2002). Tempo and mode in evolution: Phylogenetic inertia, adaptation and comparative methods. Journal of Evolutionary Biology, 15, 899-910.

Blomberg, S. P., Garland, T., \& Ives, A. R. (2003). Testing for phylogenetic signal in comparative data: Behavioral traits are more labile. Evolution, 57, 717-745.

Boesch, C., \& Boesch-Achermann, H. (2000). The chimpanzees of the Tai Forest. Oxford: Oxford University Press.

Capellini, I., Barton, R. A., McNamara, P., Preston, B., \& Nunn, C. L. (2008a). Ecology and Evolution of Mammalian Sleep. Evolution, 62, 1764-1776.

Capellini, I., Nunn, C. L., McNamara, P., Preston, B., \& Barton, R. A. (2008b). Sleep cycles, predators and energetics in mammals. Functional Ecology, 22, 847-853.

Carroll, D. A., Denenberg, V. H., \& Thoman, E. B. (1999). A comparative study of quiet sleep, active sleep, and waking on the first 2 days of life. Developmental Psychobiology, 35(1), 43-48.

Carskadon, M. A., \& Dement, W. C. (2006). Normal human sleep: An overview. In M. H. Kryger, T. Roth, \& W. C. Dement (Eds.), Principles and practice of sleep medicine $\left(4^{\text {th }}\right.$ ed., pp. 13-23). Philadelphia: WB Saunders.

Cartmill, M. (1974). Rethinking primate origins. Science, 184(135), 436-443.

Chapman, C. A., Gillespie, T. R., \& Goldberg, T. L. (2005). Primates and the ecology of their infectious diseases: How will anthropogenic change affect host-parasite interactions? Evolutionary Anthroplogy, 14,134-144.

Corbet, G. B., \& Hill, J. E. (1991). A world list of mammalian species. Oxford: Oxford University Press. 
Davies, C. R., Ayres, J. M., Dye, C., \& Deane, L. M. (1991). Malaria infection rate of Amazonian primates increases with body weight and group size. Functional Ecology 5, $655-662$.

Day, R. T., \& Elwood, R. W. (1999). Sleeping site selection by the golden-handed tamarin Saguinus midas midas: The role of predation risk, proximity to feeding sites, and territorial defence. Ethology, 105,1035-1051.

Di Bitetti, M. S., Vidal, E. M. L., Baldovino, M. C., \& Benesovsky, V. (2000). Sleeping site preferences in tufted capuchin monkeys (Cebus apella nigritus). American Journal of Primatology, 50, 257-274.

Disotell, T. R. (2008). Primate Phylogenetics. In: Encyclopedia of Life Sciences. Chinchester: John Wiley and Sons, Ltd.

Dunbar, R. I. M. (1992). Neocortex size as a constraint on group size in primates. Journal of Human Evolution, 20, 469-493.

Dunbar, R. I. M. (1998). The social brain hypothesis. Evolutionary Anthropology, 6, 178-190.

Elgar, M. A., Pagel, M. D., \& Harvey, P. H. (1988). Sleep in Mammals. Animal Behavior, 36, 1407-1419.

Felsenstein, J. (1985). Phylogenies and the comparative method. The American Naturalist, 125, $1-15$.

Foley, R. A., \& Lee, P. C. (1989). Finite social space, evolutionary pathways, and reconstructing hominid behavior. Science, 243, 901-906.

Freckleton, R. P., Harvey, P. H., \& Pagel, M. (2002). Phylogenetic analysis and comparative data: A test and review of evidence. The American Naturalist, 160, 712-726. 
Fruth, B., \& Hohmann, G. (1993). Comparative analyses of nest building behavior in bonobos and chimpanzees. In R. W. Wrangham, W. C. McGrew, F. B. M. de Waal, \& P. G. Heltne (Eds.), Chimpanzee Cultures (pp. 109-128). Cambridge, MA: Harvard University Press.

Ganguly-Fitzgerald, I., Donlea, J., \& Shaw, P. J. (2006). Waking experience affects sleep need in Drosophila. Science, 313, 1775-1781.

Garland, T., Harvey, P. H., \& Ives, A. R. (1992). Procedures for the analysis of comparative data using phylogenetically independent contrasts. Systematic Biology, 4, 18-32.

Gartlan, J. S., \& Brain, C. K. (1968). Ecology and social variability in Cercopithecus aethiops and C. mitis. In P. C. Jay (Ed.), Primates: Studies in Adaptation and Variability (pp. 253292). New York: Holt, Rinehart and Winston.

Gaulin, S. J. C., \& Gaulin, C. K. (1982). Behavioral ecology of Alouatta seniculus in Andean cloud forest. International Journal of Primatology, 3(1), 1-32.

Goodall, A. (1979). The Wandering Gorillas. London: William Collins \& Sons.

Hausfater, G., \& Meade, B. J. (1982). Alternation of sleeping groves by yellow baboons (Papio cynocephalus) as a strategy for parasite avoidance. Primates, 23, 287-297.

Heymann, E. W. (1995). Sleeping habits of tamarins, Saguinus mystax and Saguinus fuscicollis (Mammalia: Primates; Callitrichidae), in north-eastern Peru. Journal of Zoology, London, 237, 211-226.

Hilton-Taylor, C. (2002). IUCN Red List of threatened species. Morges: IUCN.

Horr, D. A. (1977). Orang-utan maturation, growing up in a female world. In S. ChevalierSkolnikoff \& F. E. Poirier (Eds.), Primate Bio-Social Development (pp. 289-321). New York: Garland Publishing. 
Hsieh, K. C., Robinson, E. L., \& Fuller, C. A. (2008). Sleep architecture in unrestrained rhesus monkeys (Macaca mulatta) synchronized to 24-hour light-dark cycles. Sleep, 31(9), $1239-1250$.

Ives, A. R., Midford, P. E., Garland, T. (2007). Within-species variation and measurement error in phylogenetic comparative methods. Systematic Biology, 56, 252-270.

Janson, C. H. (1992). Evolutionary ecology of primate social structure. In E. A. Smith, \& B. Winterhalder (Eds.), Evolutionary ecology and human behavior (pp. 95-130). New York, NY: Aldine de Gruyter.

Korstjens, A. H., Lehmann, J. \& Dunbar, R. I. M. (in review). Resting time as an ecological constraint on primate biogeography. Behavioral Ecology and Sociobiology.

Koyama, N. (1973). Dominance, grooming, and clasped-sleeping relationships among bonnet monkeys in India. Primates, 14, 225-244.

Kummer, H. (1968). Social organization of Hamadryas Baboons: A field study. Bibliotheca Primatologica, 6 .

Lesku, J. A., Roth II, T. C., Amlaner, C. J., Lima, S. L. (2006). A phylogenetic analysis of sleep architecture in mammals: The intergration of anatomy, physiology, and ecology. The American Naturalist, 168, 1-13.

Lima, S. L., Rattenborg, N. C., Lesku, J. A., \& Amlaner, C. J. (2005). Sleeping under the risk of predation. Animal Behavior, 70, 723-726.

Lindenfors, P., Nunn, C. L., \& Barton, R. A. (2007). Primate brain architecture and selection in relation to sex. BioMed Central Biology, 5 (20). doi:10.1186/1741-7007-5-20.

Martin, R. D. (1990). Primate origins and evolution. London: Chapman \& Hall. 
Martin, R. D. \& Ross, C. F. (2005). The evolutionary and ecological context of primate vision. In J. Kremers (Ed.), The primate visual system: A comparative approach (pp. 1-36). Chichester: Wiley and Sons.

McNamara, P., Capellini, I., Harris, E., Nunn, C. L., Barton, R. A., \& Preston, B. (2008). The phylogeny of sleep database: A new resource for sleep scientists. The Open Sleep Journal, 1, 11-14.

Nunn, C. L., \& Altizer, S. (2005). The global mammal parasite database: An online resource for infectious disease records in wild primates. Evolutionary Anthroplogy, 14, 1-2.

Nunn, C. L., \& Altizer, S. M. (2006). Infectious diseases in primates: Behavior, ecology and evolution. Oxford: Oxford University Press.

Nunn, C. L., \& Barton, R. A. (2001). Comparative methods for studying primate adaptation and allometry. Evolutionary Anthroplogy, 10, 81-98.

Nunn, C. L., \& Heymann, E. W. (2005). Malaria infection and host behaviour: A comparative study of Neotropical primates. Behavioral Ecology and Sociobiology, 59, 30-37.

Nunn, C. L., \& van Schaik, C. P. (2002). Reconstructing the behavioral ecology of extinct primates. In J. M. Plavcan, R. F. Kay, W. L. Jungers, \& C. P. van Schaik (Eds.), Reconstructing behavior in the fossil record (pp. 159-216). New York: Kluwer Academic/ Plenum.

Pagel, M. (1997). Inferring evolutionary processes from phylogenies. Zoologica Scripta, 26, $331-348$.

Pagel, M. (1999). Inferring the historical patterns of biological evolution. Nature, 401, 877-884.

Pagel, M., \& Meade, A. (2007). BayesTraits Version 1.0. http://www.evolution.rdg.ac.uk. Reading, UK. 
Pagel, M., Meade, A., \& Barker, D. (2004). Bayesian estimation of ancestral character states on phylogenies. Systematic Biology, 53, 673-684.

Perachio, A. A. (1971). Sleep in the nocturnal primate, Aotus trivirgatus. Proceedings of the $3^{\text {rd }}$ International Congress on Primates (vol. 2, pp. 54-60). Basel: Karger.

Plavcan, J. M., \& van Schaik, C. P. (1997). Intrasexual competition and body weight dimorphism in anthropoid primates. American Journal of Physical Anthropology, 103, 37-68.

Purvis, A. (1995). A composite estimate of primate phylogeny. Philosophical Transactions of the Royal Society, London, Series B, 348, 405-421.

Purvis, A., Nee, S., \& Harvey, P. H. (1995). Macroevolutionary inferences from primate phylogeny. Proceeding of the Royal Society London Series B, 260, 329-333.

Rattenborg, N. C., Voiren, B., Vyssotski, A. L., Kays, R. W., Spoelstra, K., Kuemmeth, F., et al. (2008). Sleeping outside the box: Electroencephalographic measues of sleep in sloths inhabiting a rainforest. The Royal Society Biology Letters. doi:10.1098/rsbl.2008.0203

Reite, M., Stynes, A. J., Vaughn, L., Pauley, J. D., \& Short, R. A. (1976). Sleep in infant monkeys: Normal values and behavioral correlates. Physiology and Behavior, 16(3), 245251.

Salzarulo, P. \& Ficca, G. (2002). Awakening and sleep-wake cycle across development. Philadelphia: J. Benjamins Pub.

Smuts, B. B., Cheney, D. L., Seyfarth, R. M., Wrangham, R. W., Struhsaker, T. T. (1987). Primate societies. Chicago, IL: Chicago University Press.

Suzuki, K. (1965). The pattern of mammalian brain gangliosides: Evaluation of the extraction procedures, postmortem changes and the effect of formalin preservation. Journal of Neurochemistry, 12(7), 629-638. 
Tattersall, I. (1987). Cathemeral activity in primates: A definition. Folia Primatologica, 49, 200202.

Thurber, A., Jha, S. K., Coleman, T., \& Frank, M. G. (2008). A preliminary study of sleep ontogenesis in the ferret (Mustela putorius furo). Behavioural Brain Research, 189, 4151.

Tobler, I. (2005). Phylogeny of sleep regulation. In M. Kryger, T. Roth, \& W. Dement (Eds.), Principles and Practice of Sleep Medicine (4 ${ }^{\text {th }}$ ed., pp. 77-90). Philadelphia, PA: W. B. Saunders.

van Schaik, C. P. (1983). Why are diurnal primates living in groups? Behaviour, 87, 120-143. van Schaik, C. P., \& Janson, C. (2000). Infanticide by males and its implications. Cambridge: Cambridge University Press.

Vessey, S. H. (1973). Night observations of free-ranging Rhesus monkeys. American Journal of Physical Anthropology, 38(2), 613-619.

Vuillon-Cacciuttolo, G., Balzamo, E., Petter, J. J., \& Bert, J. (1976). Wakefulness-sleep cycle studied by telementry in a lemurian (Lemur macaco fulvus). Revue d'électroencéphalographie et de neurophysiologie clinique, 6(1), 34-36.

Wilson, D. E., \& Reeder, D. M. (2005). Mammal species of the world. Baltimore, MD: Johns Hopkins University Press.

Wolfe, N. D., Escalante, A. A., Karesh, W. B., Kilbourn, A., Spielman, A., Lal, A. A. (1998). Wild primate populations in emerging infectious disease research: The missing link? Emerging Infectious Diseases, 4, 149-158. 
Zepelin, H., Siegel, J. M., \& Tobler, I. (2005). Mammalian sleep. In M. H. Kryger, T. Roth, \& W. C. Dement (Eds.). Principles and practice of sleep medicine (4th ed, pp. 91-100). New York, NY: W. B. Saunders. 
Table 1. Sleep quotas in primates

\begin{tabular}{|c|c|c|c|c|}
\hline Species & Total Sleep & $\begin{array}{c}\text { REM } \\
\text { Duration }\end{array}$ & $\begin{array}{c}\text { NREM } \\
\text { Duration }\end{array}$ & $\begin{array}{c}\text { Sleep cycle } \\
\text { length }\end{array}$ \\
\hline Aotus trivirgatus ${ }^{*}$ & 17 & 1.82 & 15.15 & \\
\hline Callithrix jacchus* & 9.5 & 1.61 & 7.9 & 50 \\
\hline Chlorocebus aethiops ${ }^{*}$ & 10.1 & 0.65 & 9.44 & \\
\hline Erythrocebus patas* & 10.9 & 0.86 & 9.99 & \\
\hline Eulemur macaco & 9.4 & & & \\
\hline Eulemur mongoz* & 11.9 & 0.72 & 11.16 & \\
\hline Homo sapiens* & 8.5 & 2.1 & 6.37 & 90 \\
\hline Macaca arctoides* & 9 & 1.38 & 7.65 & 50 \\
\hline Macaca mulatta* & 10.2 & 2.05 & 8.19 & \\
\hline Macaca nemestrina* & 14 & 0.92 & 13 & 80 \\
\hline Macaca radiata* & 9.1 & 1.05 & 8.06 & \\
\hline Macaca sylvanus* & 11.7 & 1.07 & 10.7 & 31.1 \\
\hline Microcebus murinus ${ }^{*}$ & 15.4 & 0.99 & 14.4 & \\
\hline Pan troglodytes* & 11.5 & 2.06 & 9.46 & 90 \\
\hline Papio anubis* & 9.2 & 1 & 8.2 & 40 \\
\hline Papio papio* & 10.1 & 1.06 & 9 & \\
\hline Perodicticus potto & 11 & & & \\
\hline Phaner furcifer & 11.5 & & & \\
\hline Saguinus oedipus & 13.2 & & & 19 \\
\hline Saimiri sciureus* & 9.7 & 1.77 & 7.8 & 12 \\
\hline Theropithecus gelada & 10.9 & & & \\
\hline
\end{tabular}

Notes: blank cells indicate that no data are available. 
Table 2. Phylogenetic Signal in Primate Sleep Parameters

\begin{tabular}{|c|c|c|}
\hline Variable & K-statistic & P-value \\
\hline Total Sleep & 0.241 & 0.108 \\
\hline NREM Sleep & 0.297 & 0.072 \\
\hline REM Sleep & 0.218 & 0.061 \\
\hline
\end{tabular}

Note: The P value does not reflect significant level of the K statistic; rather, it indicates whether the mean square error (MSE) for the data set is significantly lower than the mean MSE on permuted data sets because such a result would indicate significant phylogenetic signal (see Blomberg et al. 2003). 
Table 3. Bayesian estimates of ancestral states and evolutionary parameters

\begin{tabular}{|c|c|c|c|c|c|c|c|}
\hline & $\begin{array}{c}\text { Log-Likelihood } \\
\text { (Harmonic } \\
\text { Mean) }\end{array}$ & Ancestral & Lower & Upper & Mean $\lambda$ & Mean $\boldsymbol{\kappa}$ & Mean $\boldsymbol{9 5 \%}$ CI \\
\hline Total sleep & -47.5 & 11.3 & 9.4 & 13.4 & 0.32 & 2.39 & 1.23 \\
\hline NREM & -38.7 & 10.0 & 7.3 & 13.2 & 0.38 & 2.42 & 1.09 \\
\hline REM & -12.1 & 1.3 & 0.7 & 1.9 & 0.57 & 1.24 & 1.26 \\
\hline
\end{tabular}

Note: Results from 10,001 samples of a Bayesian posterior probability distribution, calculated in BayesTraits (Pagel and Meade, 2007). Ancestral value reflects the mean estimate, and lower and upper 95\% confidence interval (CI) reflects the distribution of values obtained from the Bayesian analysis. 


\section{Figure Legends}

Figure 1. The phylogenetic distribution of total sleep time in primates. Darker branches represent longer sleep durations per 24-hour period. Internal nodes were reconstructed using maximum parsimony. Note that the maximum parsimony reconstruction of the root node (11.8) is slightly higher (but within the confidence interval) of the estimate from the Bayesian analysis (11.3, see text and Table 2).

Figure 2. The phylogenetic distribution of NREM sleep in primates. Darker branches represent increased duration of NREM sleep. See Table 1 for details. Internal nodes were reconstructed using maximum parsimony.

Figure 3. The phylogenetic distribution of REM sleep in primates. Darker branches represent increased duration of REM sleep. See Table 1 for details. Internal nodes were reconstructed using maximum parsimony.

Figure 4. Relationships among sleep states and total sleep. Total sleep shows is highly correlated with NREM sleep (a), but shows no obvious association with REM sleep in primates (b), suggesting that NREM accounts for most of the variation in total sleep. Results for NREM and REM are, respectively: $\mathrm{t}_{14}=23.9, \mathrm{P}<0.0001 ; \mathrm{t}_{14}=0.07, \mathrm{P}=0.94$. (c) NREM and REM sleep durations are not significantly correlated $\left(\mathrm{t}_{14}=-0.51, \mathrm{p}=0.62\right)$. Analyses were based on independent contrasts calculated with the PDAP module (Midford et al., 2005) in Mesquite (Maddison and Maddison, 2006), and using Nee transformed branch lengths (Purvis, 1995) to 
better meet the assumptions of independent contrasts (Garland et al., 1992). The phylogeny matches that used in other studies of sleep in mammals (Capellini et al., 2008a).

Figure 5. Malaria prevalence in Neotropical primates in relation sleeping behavior. Mean prevalence is lower among genera that sleep in closed microhabitats in non-phylogenetic tests (Nunn and Heymann, 2005). Results were also significant in the majority of phylogenetic tests using independent contrasts, although analyses were based on only two or three evolutionary transitions depending on the phylogeny used.

Figure 6. Sampling gaps in primates. Species that have been studied for sleep are indicated with arrows next to terminal tips from a recent estimate of mammalian phylogeny (BinindaEmonds et al., 2007). Species with arrows, in order from top to bottom, are Cercopithecus aethiops, Erythrocebus patas, Papio hamadryas (representing the two species of Papio in our dataset), Theropithecus gelada, Macaca arctoides, M. radiata, M. mulatta, M. nemestrina, $M$. sylvanus, Homo sapiens, Pan troglodytes, Aotus trivirgatus, Callithrix jacchus, Saguinus oedipus, Saimiri sciureus, Microcebus murinus, Phaner furcifer, Eulemur macaco, E. mongoz, and Perodicticus potto. Not all of these species have been studied using EEG. 